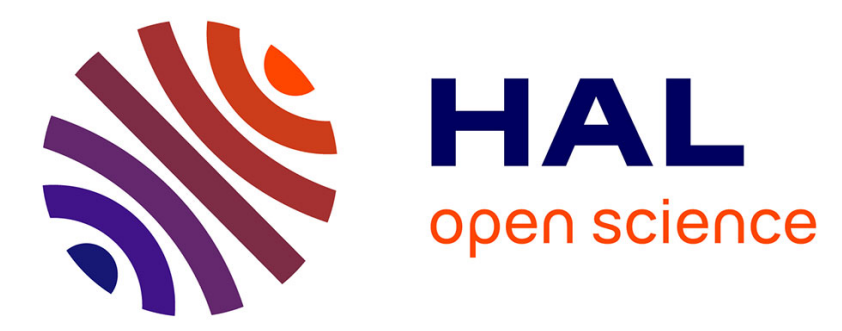

\title{
Damping of Standing Spin Waves in Bismuth-Substituted Yttrium Iron Garnet as Seen via the Time-Resolved Magneto-Optical Kerr Effect
}

Marwan Deb, Elena Popova, Michel Hehn, Niels Keller, S. Petit-Watelot, Matias Bargheer, Stéphane Mangin, Grégory Malinowski

\section{To cite this version:}

Marwan Deb, Elena Popova, Michel Hehn, Niels Keller, S. Petit-Watelot, et al.. Damping of Standing Spin Waves in Bismuth-Substituted Yttrium Iron Garnet as Seen via the Time-Resolved MagnetoOptical Kerr Effect. Physical Review Applied, 2019, 12 (4), 10.1103/PhysRevApplied.12.044006. hal-02337703

\section{HAL Id: hal-02337703 \\ https://hal.univ-lorraine.fr/hal-02337703}

Submitted on 29 Oct 2019

HAL is a multi-disciplinary open access archive for the deposit and dissemination of scientific research documents, whether they are published or not. The documents may come from teaching and research institutions in France or abroad, or from public or private research centers.
L'archive ouverte pluridisciplinaire HAL, est destinée au dépôt et à la diffusion de documents scientifiques de niveau recherche, publiés ou non, émanant des établissements d'enseignement et de recherche français ou étrangers, des laboratoires publics ou privés. 


\title{
Damping of Standing Spin Waves in Bismuth-Substituted Yttrium Iron Garnet as Seen via the Time-Resolved Magneto-Optical Kerr Effect
}

\author{
Marwan Deb®, ${ }^{1,2,{ }^{*}}$ Elena Popova, ${ }^{3}$ Michel Hehn, ${ }^{1}$ Niels Keller, ${ }^{3}$ Sébastien Petit-Watelot, ${ }^{1}$ \\ Matias Bargheer, ${ }^{2}$ Stéphane Mangin, ${ }^{1}$ and Gregory Malinowski ${ }^{1}$ \\ ${ }^{1}$ Institut Jean Lamour (IJL), CNRS UMR 7198, Université de Lorraine, 54506 Vandøuvre-lès-Nancy, France \\ ${ }^{2}$ Institut für Physik und Astronomie, Universität Potsdam, Karl-Liebknecht-Str. 24-25, 14476 Potsdam, Germany \\ ${ }^{3}$ Groupe d'Etude de la Matière Condensée (GEMaC), CNRS UMR 8635, Université de Paris-Saclay, \\ 78035 Versailles, France
}

(Received 12 June 2019; revised manuscript received 10 September 2019; published 3 October 2019)

\begin{abstract}
We investigate spin-wave resonance modes and their damping in insulating thin films of bismuthsubstituted yttrium iron garnet by performing femtosecond magneto-optical pump-probe experiments. For large magnetic fields in the range below the magnetization saturation, we find that the damping of high-order standing spin-wave (SSW) modes is about 40 times lower than that for the fundamental one. The observed phenomenon can be explained by considering different features of magnetic anisotropy and exchange fields that, respectively, define the precession frequency for fundamental and high-order SSWs. These results provide further insight into SSWs in iron garnets and may be exploited in many new photomagnonic devices.
\end{abstract}

DOI: 10.1103/PhysRevApplied.12.044006

\section{INTRODUCTION}

One of the ultimate goals of spintronics and magnonics is to use collective and coherent spin oscillations, known as spin waves (SWs) or magnons, to carry information in future information processing technologies [1-5]. Indeed, due to their low-energy dissipation, wide high-frequency spectrum (from $\mathrm{GHz}$ to $\mathrm{THz}$ ), and tunable wavelengths down to the nanoscale, SWs promote the realization of more energy efficient, extremely flexible, and faster information processing nanotechnologies compared with that of present charge-based semiconductor-based technology [1-5]. Towards that goal, intense research activity is being carried out for generating and manipulating SWs, as well as understanding the mechanism of their relaxation towards equilibrium due to damping. Traditionally, the investigation of SWs and their damping are performed using ferromagnetic resonance (FMR) techniques [6,7]. Alternatively, femtosecond laser pulses have been recently presented as an important tool to trigger SWs in a wide variety of conducting [8-10], semiconducting [11-15], and insulating [16-21] magnetic materials, as well as probing their real-time dynamics with a very high spatiotemporal resolution using femtosecond magneto-optical pump-probe techniques [22-26]. These highly resolved spatiotemporal investigations provide important opportunities to improve the understanding of SWs, with the aim

\footnotetext{
*madeb@uni-potsdam.de
}

of manipulating their dynamics at the fastest speed and in the most efficient way.

A challenging problem for high-speed applications is the generation of SWs that combine high frequency with low magnetic damping at weak external magnetic field $\left(H_{\text {ext }}\right)$. To satisfy the high-frequency requirement, exchange standing SW (SSW) modes are particularly interesting. Indeed, they are characterized by quantized wave-vectors, which are defined as $k=n \pi / d$, and precession frequencies proportional to $D_{\mathrm{ex}} k^{2}[27,28]$, where $n$ is the mode number, $d$ is the film thickness, and $D_{\text {ex }}$ is the exchange stiffness. Therefore, in nanoscale magnetic devices, high-order SSW modes can possess very high frequencies. However, exciting SSWs in nanoscale thin magnetic films with conventional microwave magnetic fields remains very complex, since it requires nanosized microwave antennas that usually have low efficiency [29]. Recently, it has been demonstrated that SSWs can be triggered by femtosecond optical excitation [8,13,30-34]. Early studies of femtosecond laser excited SSWs were performed in metallic and semiconductor magnetic materials. On the other hand, materials with low intrinsic damping, very large magneto-optical Faraday effects, and good transparency in the visible and infrared region, such as the magnetic insulator of Bi-substituted iron garnets (Bi-YIG) [35-39], are of utmost relevance for new photomagnetic applications. Therefore, exploring SSWs and their damping at low $H_{\text {ext }}$ in nanoscale Bi-YIG is of prime importance for both future SW applications and 

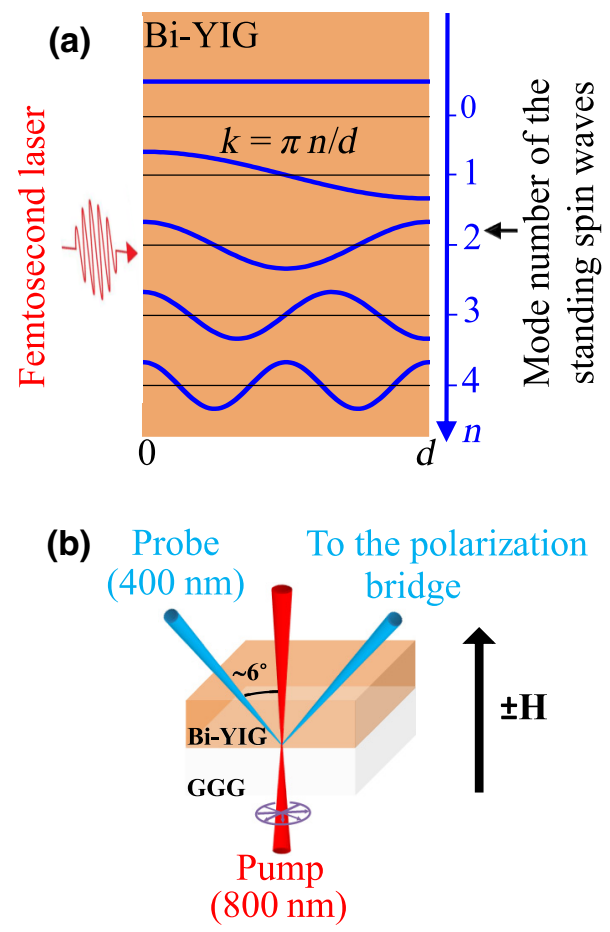

FIG. 1. (a) Schematic illustration of the femtosecond laser excitation-driven standing spin waves in Bi-YIG. (b) Sketch of the time-resolved magneto-optical Kerr effect (TR MOKE) configuration.

a fundamental understanding of ultrafast magnetization dynamics.

Here, we report an experimental investigation of SSWs triggered by femtosecond laser pluses in a nanoscale thin film of Bi-YIG with high in-plane magnetic anisotropy. We show that femtosecond laser pulses trigger highfrequency even and odd SSWs, as illustrated schematically in Fig. 1(a). We demonstrate that the damping of highorder SSW modes is about 40 times lower than that for the fundamental one for a broad applied magnetic field range below the magnetization saturation field. This behavior can be understood by considering the different features of magnetic anisotropy and exchange fields that define the precession frequency for fundamental and high-order SSWs, respectively. Moreover, we demonstrate that the damping of all SSWs is not affected by the pump energy density.

\section{SAMPLE PROPERTIES AND EXPERIMENTAL METHODS}

Our study is based on a 135-nm thick Bi-YIG film with a composition of $\mathrm{Bi}_{1} \mathrm{Y}_{2} \mathrm{Fe}_{5} \mathrm{O}_{12}$. The Bi-YIG materials are ferrimagnetic insulators that crystallize in the cubic garnet structure (space group $I a 3 d$ ), which is characterized by three crystallographic sites (tetrahedral $24 d$, octahedral $16 a$, dodecahedral 24c) $[35,37]$. The iron atoms are distributed over 24 tetrahedral and 16 octahedral nonequivalent sites, which are coupled by a strong antiferromagnetic superexchange, forming a rigid ferrimagnet with a high Curie temperature $\left(T_{C}>550 \mathrm{~K}\right)[35-37,40]$. The nonmagnetic Bi and $\mathrm{Y}$ ions occupy the dodecahedral sites. In particular, the existence of $\mathrm{Bi}$ atoms in the dodecahedral site gives arises to huge increases of the magneto-optical (MO) effect [36,37]. The investigated sample is grown by pulsed laser deposition on a (100) oriented gadolinium gallium garnet (GGG) substrate [41]. In situ ellipsometry and reflection high-energy electron diffraction measurements are performed during growth to monitor the optical constant, the growth mode, and the surface structure. After growth, an ex situ X-ray diffraction study is used to characterize the crystallographic structure. The film is a single phase with excellent crystallinity. The static magnetic and MO properties of the film are characterized using a static MO spectrometer. The out-of-plane saturation field $(\sim 260 \mathrm{mT})$ is about 50 times larger than the in-plane saturation field $(\sim 5 \mathrm{mT})$; this reveals a strong in-plane magnetic anisotropy. This in-plane easy axis magnetization is due to a strong negative growth-induced uniaxial anisotropy field, $H_{u}[42,43]$, which is, in our film, about $-83 \mathrm{mT}$. On the other hand, the polar Faraday and Kerr MO spectra of the $\mathrm{Bi}_{1} \mathrm{Y}_{2} \mathrm{Fe}_{5} \mathrm{O}_{12}$ film are consistent with previous studies of MO properties in Bi-YIG [36,44]. The laser-induced ultrafast spin dynamics is investigated at room temperature by TR MOKE using the configuration sketched in Fig. 1(b). Briefly, an amplified Ti:sapphire laser system operating at $5-\mathrm{kHz}$ repetition rate and delivering 35-fs laser pulses is used to generate the pump and probe beams. The pump beam is kept at the fundamental wavelength of the amplifier at $800 \mathrm{~nm}$, while the probe beam is frequency-doubled to $400 \mathrm{~nm}$. Both beams are linearly polarized and focused on the sample down to a spot size of about $60 \mu \mathrm{m}$ for the probe and around $330 \mu \mathrm{m}$ for the pump. The probe beam reflected by the Bi-YIG layer allows differential changes of the polar Kerr rotation, $\Delta \Theta_{K}(t)$, to be measured as a function of the time delay. The external magnetic field, $H_{\text {ext }}$, is applied perpendicular to the plane of the film.

\section{RESULTS AND DISCCUSSION}

Figure 2(a) shows the TR MOKE induced by a pump energy density of $E_{\text {pump }}=12.47 \mathrm{~mJ} \mathrm{~cm}^{-2}$ for $H_{\text {ext }}= \pm 66 \mathrm{mT}$. It displays spin dynamics with complicated oscillations, which are formed by five resonance modes, as revealed by the FFT shown in Fig. 2(b). The magnetic nature of these resonance modes is clearly demonstrated by the sign change of the TR MOKE signal with reversal of the direction of $H_{\text {ext }}$. Interestingly, the precession frequencies of these modes have a quadratic dependence on the mode number [Fig. 2(c)]. Such variation can be well described by the dispersion relation 


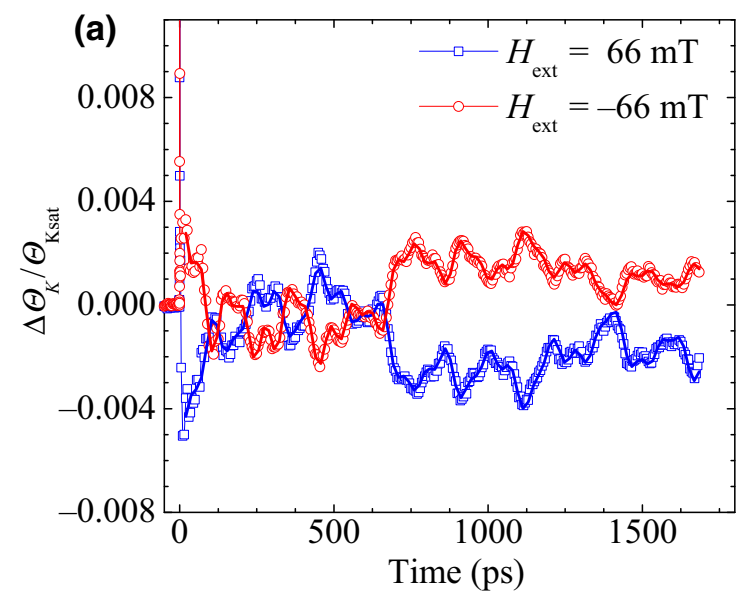

(b)

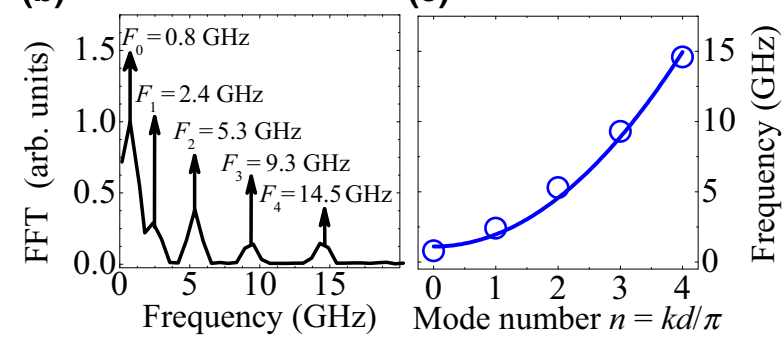

FIG. 2. Laser-induced standing spin waves in the garnet sample. (a) $\Delta \Theta_{K} / \Theta_{\text {Ksat }}$ induced by a pump energy density of $12.47 \mathrm{~mJ} \mathrm{~cm}^{-2}$ for $H_{\text {ext }}= \pm 66 \mathrm{mT}$. The solid lines are the fits to the data using Eq. (2). (b) Fast Fourier transform (FFT) spectrum of the $\Delta \Theta_{K} / \Theta_{\text {Ksat }}$ signal. (c) Spin precession frequency as a function of the mode number. The solid line is the fit obtained using Eq. (1).

of SSWs [27,28], which is written, in the case of our experimental configuration, as [27,28]:

$$
\omega(k)=\omega_{0}+\gamma D_{\mathrm{ex}} k^{2}
$$

where $\omega$ is the angular precession frequency; $\omega_{0}$ is the angular frequency of the fundamental mode, which depends on $H_{\text {ext }}$ and the effective magnetic anisotropy field; $\gamma$ is the gyromagnetic ratio; $D_{\mathrm{ex}}$ is the exchange stiffness; and $k=n \pi / d$ represents the wave-vector of the standing spin waves given by $d=135 \mathrm{~nm}$. Indeed, a fit of experimental data with Eq. (1) using $(\gamma / 2 \pi)=28 \mathrm{GHz} / \mathrm{T}$ yields an excellent agreement with the experimental results [Fig. 2(c)]. We obtain an exchange stiffness of $D_{\mathrm{ex}}=(5.9 \pm 0.2) \quad 10^{-17} \mathrm{~T} \mathrm{~m} \mathrm{~m}^{2}$, which is in good agreement with those reported in the literature for YIG and Bi-YIG [45-47]. This clearly demonstrates the SSW nature of the observed magnetic resonances modes. We note that the characteristics of the excited SSWs, such as the amplitude and phases, are independent of the polarization of the pump beam. This reveals that pump polarization-dependent mechanisms, such as the inverse Cotton-Mouton effect [48-50], is not at the origin of the excitation. The excitation of the SSWs is related to an ultrafast change of magnetic anisotropy induced by incoherent and coherent phonons due to the temperature dependence of the magnetic anisotropy constants [51,52] and inverse magnetostriction [53-56], respectively. The coherent and incoherent phonons are generated via the absorption of the pump photons by the phonon-assisted electronic d-d transitions simultaneously by one- and two-photon absorption processes [56].

To study the properties of the SSWs in more detail, TR MOKE measurements are fitted with the following timedependent damped oscillators:

$$
\Delta \Theta_{K}(t)=\sum_{i=0}^{4} A_{i} e^{-\left(t / \tau_{i}\right)} \sin \left(2 \pi f_{i} t-\phi_{i}\right)+B e^{-C t},
$$

where $A_{i}, f_{i}, \phi_{i}$, and $\tau_{i}$ are, respectively, the amplitude, frequency, initial phase, and decay time characterizing the precession of mode $i(i=0,1,2,3$, and 4$)$. The term $B e^{-C t}$ represents a $\mathrm{MO}$ background signal. The corresponding fitting curve obtained with Eq. (2) is plotted in Fig. 2(a), with solid lines showing a good agreement with the experimental data. We can thus extract precession frequencies of $f_{0}=0.8 \mathrm{GHz}, f_{1}=2.4 \mathrm{GHz}, f_{2}=5.3 \mathrm{GHz}, f_{3}=9.3 \mathrm{GHz}$ and $f_{4}=14.5 \mathrm{GHz}$, which are identical to those obtained by FFT analysis. These adjustments also allow the effective damping of each SSW mode to be extracted, which can be defined as $\alpha_{i}=\left(1 / f_{i} \tau_{i}\right)$. Interestingly, we find that the effective damping decreases by more than 40 times upon increasing the mode number (see Fig. 3). At large mode numbers, it converges to the intrinsic damping value of $\alpha \approx 5 \times 10^{-3}$, which characterizes the precession of the uniform mode $(n=0)$ at high $H_{\text {ext }}$ above approximately $450 \mathrm{mT}$, as measured by the FMR technique.

To provide a comprehensive understanding of the behavior of the damping coefficient with the mode number,

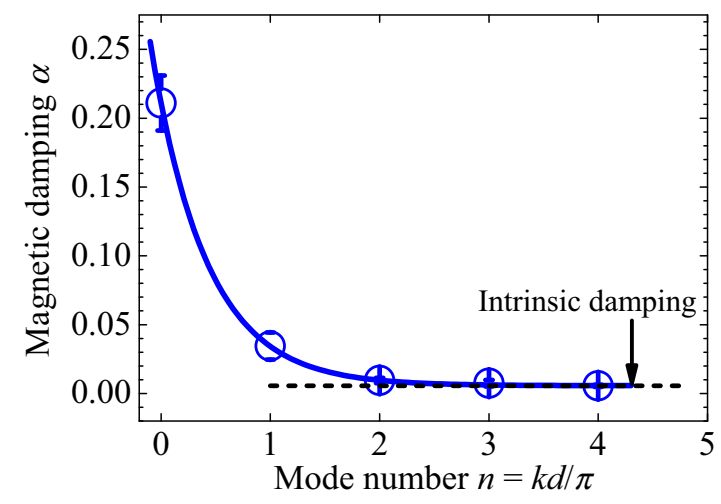

FIG. 3. Variation of the magnetic damping as a function of the standing spin-wave mode number measured for $H_{\mathrm{ext}}=66 \mathrm{mT}$ and a pump energy density of $12.47 \mathrm{~mJ} \mathrm{~cm}^{-2}$. 

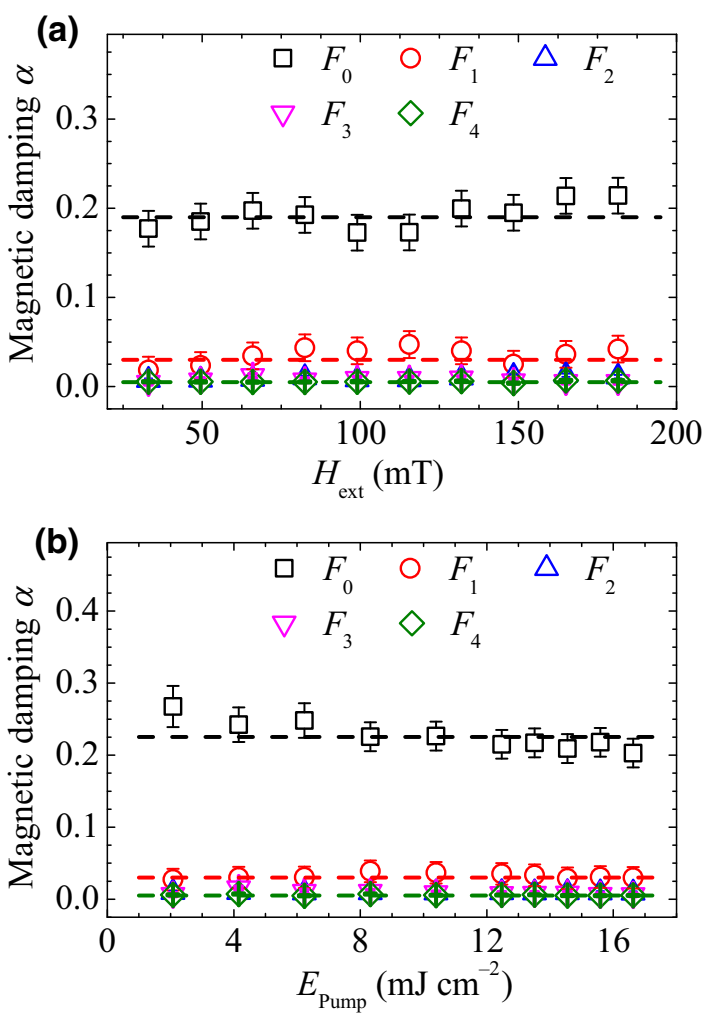

FIG. 4. Magnetic damping of standing spin-wave modes as a function of (a) the external magnetic field and (b) the pump energy density.

we recall some fundamentals of magnetization precession and damping in magnetic media. The uniform spinwave mode has been extensively investigated in various magnetic materials $[13,14,30,57]$. In particular, it is demonstrated that damping of the uniform mode usually increases with decreasing $H_{\text {ext }}$ below the saturation magnetization field, $H_{\text {sat }}$, where the precession frequency is mainly defined by the magnetic anisotropy field. The phenomenon is attributed to decoherence of the precession, which can originate from a spread of excited frequencies of the uniform mode due to a weak inhomogeneity in the magnetic anisotropy field [30]. On the other hand, contrary to the uniform mode, the precession frequency of high-order SSWs is mainly defined by the exchange field, $H_{\text {ex }}=D_{\text {ex }} k^{2}$ [see Eq. (1)], which is zero for the fundamental mode. From a microscopic point of view, the exchange stiffness, $D_{\text {ex }}$, is directly related to the exchange interaction constants, $J_{i j}$ between the Fe magnetic moments [58], which are determined by the local $\mathrm{Fe}-\mathrm{Fe}$ and $\mathrm{Fe}-\mathrm{O}$ distances $[59,60]$. Due to very good crystallinity of the studied sample, together with the well-known high rigidity of the garnet structure [35], $H_{\mathrm{ex}}$ should be almost perfectly homogeneous across the whole Bi-YIG film. This ensures a good coherence of the precession frequency for highorder SSWs, limiting the magnetic losses, and therefore, reducing the Gilbert damping down to its intrinsic value (Fig. 3).

To further investigate the damping of SSWs, we perform TR MOKE measurements as a function of the external magnetic field and pump energy density. The extracted damping coefficients obtained by analyzing the TR MOKE data with Eq. (2) are shown in Fig. 4. The high-order SSWs retain a very low damping value for a $H_{\text {ext }}$ value of only $33 \mathrm{mT}$. This is in good agreement with the expected homogeneity of the exchange field in iron garnets. We also show that the damping of the fundamental mode is almost constant for a large $H_{\text {ext }}$ range below that of the saturation magnetization. On the other hand, we find that the damping of all SSW mode do not depend on $E_{\text {pump. This }}$ behavior is different from that associated with SWs triggered by femtosecond laser pulses in metallic ferro- and ferrimagnets due to their high absorption, which drives an increase of the sample temperature and damping [61, 62]. This behavior in metallic magnets is qualitatively in agreement with theoretical studies that predict an increase in damping, when the static temperature of the sample increases towards $T_{C}[63,64]$. However, in dielectric iron garnet, the increase of the sample temperature induced by a $800 \mathrm{~nm}$ pump pulse with $E_{\text {pump }}=20 \mathrm{~mJ} \mathrm{~cm}^{-2}$ cannot exceed $10 \mathrm{~K}[52,56,65]$ and the sample temperature remains very far from $T_{C}$. This is reflected by no, or only very low, dependence of the damping on $E_{\text {pump }}$, which is an additional advantage of laser-induced SWs in magnetic insulators.

\section{CONCLUSION}

We study the laser-induced standing spin waves in bismuth iron garnet with high in-plane magnetic anisotropy. We show that femtosecond laser pulses trigger highfrequency even and odd SSW modes. We discover that the effective damping of high-order SSW modes is about 40 times lower than that of the fundamental one for magnetic fields below the magnetization saturation field. We explain this phenomenon by considering different features of the magnetic anisotropy and exchange fields that, respectively, define the precession frequency for fundamental and highorder SSWs. Moreover, we demonstrate that the effective damping for all SSWs does not depend on the pump energy density. These findings highlight the suitability of a femtosecond laser pulse for triggering SSWs that combine high frequency and low damping at weak magnetic field, and open up interesting prospects for new photomagnonic devices.

\section{ACKNOWLEDGMENTS}

This work is supported by the ANR-NSF Project Grant No. ANR-15-CE24-0009 UMAMI, the Alexander von Humboldt Foundation, and the BMBF Project Grant No. 05K16IPA. Experiments are performed using equipment 
from the TUBE Dépôt et Analyse sous Ultravide de nano Matériaux funded by FEDER (EU), ANR, Région Grand Est, and Metropole Grand Nancy.

[1] A. A. Serga, A. V. Chumak, and B. Hillebrands, YIG magnonics, J. Phys. D: Appl. Phys. 43, 264002 (2010).

[2] B. Lenk, H. Ulrichs, F. Garbs, and M. Münzenberg, The building blocks of magnonics, Phys. Rep. 507, 107 (2011).

[3] A. V. Chumak, A. A. Serga, and B. Hillebrands, Magnon transistor for all-magnon data processing, Nat. Commun. 5, 4700 (2014).

[4] A. V. Chumak, V. I. Vasyuchka, A. A. Serga, and B. Hillebrands, Magnon spintronics, Nat. Phys. 11, 453 (2015).

[5] D. Grundler, Reconfigurable magnonics heats up, Nat. Phys. 11, 438 (2015).

[6] M. Farle, Ferromagnetic resonance of ultrathin metallic layers, Rep. Prog. Phys. 61, 755 (1998).

[7] B. Heinrich, in Ultrathin Magnetic Structures III: Fundamentals of Nanomagnetism, edited by J. A. C. Bland, B. Heinrich (Springer Berlin Heidelberg, Berlin, Heidelberg, 2005).

[8] M. van Kampen, C. Jozsa, J. T. Kohlhepp, P. LeClair, L. Lagae, W. J. M. de Jonge, and B. Koopmans, All-Optical Probe of Coherent Spin Waves, Phys. Rev. Lett. 88, 227201 (2002).

[9] M. Vomir, L. H. F. Andrade, L. Guidoni, E. Beaurepaire, and J. Y. Bigot, Real Space Trajectory of the Ultrafast Magnetization Dynamics in Ferromagnetic Metals, Phys. Rev. Lett. 94, 237601 (2005).

[10] C. D. Stanciu, A. V. Kimel, F. Hansteen, A. Tsukamoto, A. Itoh, A. Kirilyuk, and T. Rasing, Ultrafast spin dynamics across compensation points in ferrimagnetic GdFeCo: The role of angular momentum compensation, Phys. Rev. B 73, 220402(R) (2006).

[11] Y. Hashimoto, S. Kobayashi, and H. Munekata, Photoinduced Precession of Magnetization in Ferromagnetic (Ga, Mn)As, Phys. Rev. Lett. 100, 067202 (2008).

[12] E. Rozkotová, P. Němec, N. Tesařová, P. Malý, V. Novák, K. Olejník, M. Cukr, and T. Jungwirth, Coherent control of magnetization precession in ferromagnetic semiconductor (Ga, Mn)As, Appl. Phys. Lett. 93, 232505 (2008).

[13] P. Němec, V. Novák, N. Tesařová, E. Rozkotová, H. Reichlová, D. Butkovičová, F. Trojánek, K. Olejník, P. Malý, R. P. Campion, B. L. Gallagher, J. Sinova, and T. Jungwirth, The essential role of carefully optimized synthesis for elucidating intrinsic material properties of (Ga, Mn)As, Nat. Commun. 4, 1422 (2013).

[14] R. R. Subkhangulov, H. Munekata, T. Rasing, and A. V. Kimel, Laser-induced spin dynamics in ferromagnetic (In, Mn)As at magnetic fields up to 7T, Phys. Rev. B 89, 060402(R) (2014).

[15] N. Tesařová, D. Butkovičová, R. P. Campion, A. W. Rushforth, K. W. Edmonds, P. Wadley, B. L. Gallagher, E. Schmoranzerová, F. Trojánek, P. Malý, P. Motloch, V. Novák, T. Jungwirth, and P. Němec, Comparison of micromagnetic parameters of the ferromagnetic semiconductors (Ga, Mn)As, P and (Ga, Mn)As, Phys. Rev. B 90, 155203 (2014).
[16] A. V. Kimel, A. Kirilyuk, P. A. Usachev, R. V. Pisarev, A. M. Balbashov, and T. Rasing, Ultrafast non-thermal control of magnetization by instantaneous photomagnetic pulses, Nature 435, 655 (2005).

[17] F. Hansteen, A. Kimel, A. Kirilyuk, and T. Rasing, Femtosecond Photomagnetic Switching of Spins in Ferrimagnetic Garnet Films, Phys. Rev. Lett. 95, 047402 (2005).

[18] A. V. Kimel, C. D. Stanciu, P. A. Usachev, R. V. Pisarev, V. N. Gridnev, A. Kirilyuk, and T. Rasing, Optical excitation of antiferromagnetic resonance in TmFeO3, Phys. Rev. B 74, 060403(R) (2006).

[19] M. Deb, M. Vomir, J.-L. Rehspringer, and J.-Y. Bigot, Ultrafast optical control of magnetization dynamics in polycrystalline bismuth doped iron garnet thin films, Appl. Phys. Lett. 107, 252404 (2015).

[20] B. Koene, M. Deb, E. Popova, N. Keller, T. Rasing, and A. Kirilyuk, Excitation of magnetic precession in bismuth iron garnet via a polarization-independent impulsive photomagnetic effect, Phys. Rev. B 91, 184415 (2015).

[21] M. Deb, P. Molho, B. Barbara, and J.-Y. Bigot, Temperature and magnetic field dependence of rare-earth $\leftrightarrow$ iron exchange resonance mode in a magnetic oxide studied with femtosecond magneto-optical Kerr effect, Phys. Rev. B 94, 054422 (2016).

[22] T. Satoh, Y. Terui, R. Moriya, B. A. Ivanov, K. Ando, E. Saitoh, T. Shimura, and K. Kuroda, Directional control of spin-wave emission by spatially shaped light, Nat. Photon. 6, 662 (2012).

[23] Y. Au, M. Dvornik, T. Davison, E. Ahmad, P. S. Keatley, A. Vansteenkiste, B. Van Waeyenberge, and V. V. Kruglyak, Direct Excitation of Propagating Spin Waves by Focused Ultrashort Optical Pulses, Phys. Rev. Lett. 110, 097201 (2013).

[24] S. Iihama, Y. Sasaki, A. Sugihara, A. Kamimaki, Y. Ando, and S. Mizukami, Quantification of a propagating spinwave packet created by an ultrashort laser pulse in a thin film of a magnetic metal, Phys. Rev. B 94, 020401(R) (2016).

[25] Y. Hashimoto, S. Daimon, R. Iguchi, Y. Oikawa, K. Shen, K. Sato, D. Bossini, Y. Tabuchi, T. Satoh, B. Hillebrands, G. E. W. Bauer, T. H. Johansen, A. Kirilyuk, T. Rasing, and E. Saitoh, All-optical observation and reconstruction of spin wave dispersion, Nat. Commun. 8, 15859 (2017).

[26] M. Jäckl, V. I. Belotelov, I. A. Akimov, I. V. Savochkin, D. R. Yakovlev, A. K. Zvezdin, and M. Bayer, Magnon Accumulation by Clocked Laser Excitation as Source of Long-Range Spin Waves in Transparent Magnetic Films, Phys. Rev. X 7, 021009 (2017).

[27] C. Kittel, Introduction to solid state physics (John Wiley \& Sons, New York, 1986).

[28] A. H. Morrish, The Physical Principles of Magnetism (IEEE, New York, 2001).

[29] H. Yu, O. d' Allivy Kelly, V. Cros, R. Bernard, P. Bortolotti, A. Anane, F. Brandl, F. Heimbach, and D. Grundler, Approaching soft X-ray wavelengths in nanomagnet-based microwave technology, Nat. Commun. 7, 11255 (2016).

[30] J. Walowski, M. D. Kaufmann, B. Lenk, C. Hamann, J. McCord, and M. Münzenberg, Intrinsic and nonlocal Gilbert damping in polycrystalline nickel studied by Ti : sapphire laser fs spectroscopy, J. Phys. D: Appl. Phys. 41, 164016 (2008). 
[31] B. Lenk, G. Eilers, J. Hamrle, and M. Münzenberg, Spinwave population in nickel after femtosecond laser pulse excitation, Phys. Rev. B 82, 134443 (2010).

[32] S. Shihab, H. Riahi, L. Thevenard, H. J. v. Bardeleben, A. Lemaitre, and C. Gourdon, Systematic study of the spin stiffness dependence on phosphorus alloying in the ferromagnetic semiconductor (Ga, Mn)As, Appl. Phys. Lett. 106, 142408 (2015).

[33] S. Shihab, L. Thevenard, A. Lemaître, and C. Gourdon, Counter-rotating standing spin waves: A magneto-optical illusion, Phys. Rev. B 95, 144411 (2017).

[34] M. Djordjevic, G. Eilers, A. Parge, M. Münzenberg, and J. S. Moodera, Intrinsic and nonlocal Gilbert damping parameter in all optical pump-probe experiments, J. Appl. Phys. 99, 08F308 (2006).

[35] G. D. Winkler, Magnetic garnets (Vieweg, Braunschweig, 1981).

[36] P. Hansen and J. P. Krumme, Magnetic and magneto-optical properties of garnet films, Thin Solid Films 114, 69 (1984).

[37] G. F. Dionne, Magnetic oxides (Springer, New York, 2009).

[38] M. Deb, E. Popova, A. Fouchet, and N. Keller, Magnetooptical Faraday spectroscopy of completely bismuthsubstituted Bi3Fe5O12 garnet thin films, J. Phys. D: Appl. Phys. 45, 455001 (2012).

[39] L. Soumah, N. Beaulieu, L. Qassym, C. Carrétéro, E. Jacquet, R. Lebourgeois, J. Ben Youssef, P. Bortolotti, V. Cros, and A. Anane, Ultra-low damping insulating magnetic thin films get perpendicular, Nat. Commun. 9, 3355 (2018).

[40] B. Vertruyen, R. Cloots, J. S. Abell, T. J. Jackson, R. C. da Silva, E. Popova, and N. Keller, Curie temperature, exchange integrals, and magneto-optical properties in offstoichiometric bismuth iron garnet epitaxial films, Phys. Rev. B 78, 094429 (2008).

[41] E. Popova, L. Magdenko, H. Niedoba, M. Deb, B. Dagens, B. Berini, M. Vanwolleghem, C. Vilar, F. Gendron, A. Fouchet, J. Scola, Y. Dumont, M. Guyot, and N. Keller, Magnetic properties of the magnetophotonic crystal based on bismuth iron garnet, J. Appl. Phys. 112, 093910 (2012).

[42] S. A. Manuilov, S. I. Khartsev, and A. M. Grishin, Pulsed laser deposited Y3Fe5O12 films: Nature of magnetic anisotropy I, J. Appl. Phys. 106, 123917 (2009).

[43] S. A. Manuilov and A. M. Grishin, Pulsed laser deposited Y3Fe5O12 films: Nature of magnetic anisotropy II, J. Appl. Phys. 108, 013902 (2010).

[44] C. Ming-Yau, L. Fang-Yuh, L. Da-Ren, Y. Kuang, and L. Juin-Sen, Red shift of faraday rotation in thin films of completely bismuth-substituted iron garnet Bi3Fe5O12, Jpn. J. Appl. Phys. 38, 6687 (1999).

[45] A. Gurevich and A. Anisimov, Intrinsic spin wave relaxation processes in yttrium iron garnets, Zh. Eksp. Teor. Fiz 68, 677 (1975).

[46] G. G. Siu, C. M. Lee, and Y. Liu, Magnons and acoustic phonons in Y3 - xBixFe5O12, Phys. Rev. B 64, 094421 (2001).

[47] S. Klingler, A. V. Chumak, T. Mewes, B. Khodadadi, C. Mewes, C. Dubs, O. Surzhenko, B. Hillebrands, and A. Conca, Measurements of the exchange stiffness of YIG films using broadband ferromagnetic resonance techniques, J. Phys. D: Appl. Phys. 48, 015001 (2014).
[48] A. M. Kalashnikova, A. V. Kimel, R. V. Pisarev, V. N. Gridnev, A. Kirilyuk, and T. Rasing, Impulsive Generation of Coherent Magnons by Linearly Polarized Light in the EasyPlane Antiferromagnet FeBO3, Phys. Rev. Lett. 99, 167205 (2007).

[49] I. Yoshimine, T. Satoh, R. Iida, A. Stupakiewicz, A. Maziewski, and T. Shimura, Phase-controllable spin wave generation in iron garnet by linearly polarized light pulses, J. Appl. Phys. 116, 043907 (2014).

[50] L. Q. Shen, L. F. Zhou, J. Y. Shi, M. Tang, Z. Zheng, D. Wu, S. M. Zhou, L. Y. Chen, and H. B. Zhao, Dominant role of inverse Cotton-Mouton effect in ultrafast stimulation of magnetization precession in undoped yttrium iron garnet films by 400-nm laser pulses, Phys. Rev. B 97, 224430 (2018).

[51] L. A. Shelukhin, V. V. Pavlov, P. A. Usachev, P. Y. Shamray, R. V. Pisarev, and A. M. Kalashnikova, Ultrafast laser-induced changes of the magnetic anisotropy in a low-symmetry iron garnet film, Phys. Rev. B 97, 014422 (2018).

[52] C. S. Davies, K. H. Prabhakara, M. D. Davydova, K. A. Zvezdin, T. B. Shapaeva, S. Wang, A. K. Zvezdin, A. Kirilyuk, T. Rasing, and A. V. Kimel, Anomalously Damped Heat-Assisted Route for Precessional Magnetization Reversal in an Iron Garnet, Phys. Rev. Lett. 122, 027202 (2019).

[53] A. V. Scherbakov, A. S. Salasyuk, A. V. Akimov, X. Liu, M. Bombeck, C. Brüggemann, D. R. Yakovlev, V. F. Sapega, J. K. Furdyna, and M. Bayer, Coherent Magnetization Precession in Ferromagnetic (Ga, Mn)As Induced by Picosecond Acoustic Pulses, Phys. Rev. Lett. 105, 117204 (2010).

[54] J.-W. Kim, M. Vomir, and J.-Y. Bigot, Ultrafast Magnetoacoustics in Nickel Films, Phys. Rev. Lett. 109, 166601 (2012).

[55] V. N. Kats, T. L. Linnik, A. S. Salasyuk, A. W. Rushforth, M. Wang, P. Wadley, A. V. Akimov, S. A. Cavill, V. Holy, A. M. Kalashnikova, and A. V. Scherbakov, Ultrafast changes of magnetic anisotropy driven by laser-generated coherent and noncoherent phonons in metallic films, Phys. Rev. B 93, 214422 (2016).

[56] M. Deb, P. Molho, B. Barbara, and J.-Y. Bigot, Controlling laser-induced magnetization reversal dynamics in a rareearth iron garnet across the magnetization compensation point, Phys. Rev. B 97, 134419 (2018).

[57] G. Counil, J.-V. Kim, T. Devolder, C. Chappert, K. Shigeto, and Y. Otani, Spin wave contributions to the highfrequency magnetic response of thin films obtained with inductive methods, J. Appl. Phys. 95, 5646 (2004).

[58] C. M. Srivastava and R. Aiyar, Spin wave stiffness constants in some ferrimagnetics, J. Phys. C: Solid State Phys. 20, 1119 (1987).

[59] W. Noun, E. Popova, F. Bardelli, Y. Dumont, R. Bertacco, A. Tagliaferri, M. Tessier, M. Guyot, B. Berini, and N. Keller, Determination of yttrium iron garnet superexchange parameters as a function of oxygen and cation stoichiometry, Phys. Rev. B 81, 054411 (2010).

[60] Y. Dumont, N. Keller, E. Popova, D. S. Schmool, M. Tessier, S. Bhattacharya, B. Stahl, R. M. C. Da Silva, and M. Guyot, Tuning magnetic properties with offstoichiometry in oxide thin films: An experiment with 
yttrium iron garnet as a model system, Phys. Rev. B 76, 104413 (2007).

[61] B. Liu, X. Ruan, Z. Wu, H. Tu, J. Du, J. Wu, X. Lu, L. He, R. Zhang, and Y. Xu, Transient enhancement of magnetization damping in $\mathrm{CoFeB}$ film via pulsed laser excitation, Appl. Phys. Lett. 109, 042401 (2016).

[62] S. Mondal and A. Barman, Laser Controlled Spin Dynamics of Ferromagnetic Thin Film from Femtosecond to Nanosecond Timescale, Phys. Rev. Appl. 10, 054037 (2018).

[63] F. Schlickeiser, U. Atxitia, S. Wienholdt, D. Hinzke, O. Chubykalo-Fesenko, and U. Nowak, Temperature dependence of the frequencies and effective damping parameters of ferrimagnetic resonance, Phys. Rev. B 86, 214416 (2012).

[64] N. A. Natekar, W.-H. Hsu, and R. H. Victora, Calculated dependence of FePt damping on external field magnitude and direction, Aip Adv. 7, 056004 (2017).

[65] B. Koene, M. Deb, E. Popova, N. Keller, T. Rasing, and A. Kirilyuk, Spectrally resolved optical probing of laser induced magnetization dynamics in bismuth iron garnet, J. Phys.: Condens. Matter 28, 276002 (2016). 